\title{
Adapting Advanced Inorganic Chemistry Lecture and Laboratory Instruction for a Legally Blind Student
}

John R. Miecznikowski

Fairfield University, jmiecznikowski@fairfield.edu

Matthew J. Guberman-Pfeffer

Elizabeth E. Butrick

Julie A. Colangelo

Cristine E. Donaruma

Follow this and additional works at: https://digitalcommons.fairfield.edu/chemistry-facultypubs Copyright 2015 American Chemical Society. Post-print has been archived here with permission from copyright holder. Final publisher version available http://pubs.acs.org/doi/abs/10.1021/ ed500489c

\section{Repository Citation}

Miecznikowski, John R.; Guberman-Pfeffer, Matthew J.; Butrick, Elizabeth E.; Colangelo, Julie A.; and Donaruma, Cristine E., "Adapting Advanced Inorganic Chemistry Lecture and Laboratory Instruction for a Legally Blind Student" (2015). Chemistry \& Biochemistry Faculty Publications. 27.

https://digitalcommons.fairfield.edu/chemistry-facultypubs/27

\section{Published Citation}

Miecznikowski, John R., Matthew J. Guberman-Pfeffer, Elizabeth E. Butrick, Julie A. Colangelo, and Cristine E. Donaruma. "Adapting Advanced Inorganic Chemistry Lecture and Laboratory Instruction for a Legally Blind Student." Journal of Chemical Education 92, no. 8 (2015): 1344-1352. DOI: 10.1021/ed500489c

This item has been accepted for inclusion in DigitalCommons@Fairfield by an authorized administrator of DigitalCommons@Fairfield. It is brought to you by DigitalCommons@Fairfield with permission from the rightsholder(s) and is protected by copyright and/or related rights. You are free to use this item in any way that is permitted by the copyright and related rights legislation that applies to your use. For other uses, you need to obtain permission from the rights-holder(s) directly, unless additional rights are indicated by a Creative Commons license in the record and/or on the work itself. For more information, please contact digitalcommons@fairfield.edu. 


\title{
Adapting Advanced Inorganic Chemistry Lecture and Laboratory Instruction for a Legally Blind Student
}

John R. Miecznikowski, * Matthew Guberman-Pfeffer, Elizabeth E. Butrick, Julie A. Colangelo, and Cristine E. Donaruma

\author{
Fairfield University \\ Department of Chemistry \& Biochemistry \\ 1073 North Benson Road \\ Fairfield, CT 06824 \\ jmiecznikowski@ffairfield.edu \\ $1-(203) 254-4000 \times 2125$
}

fax: 1-(203) 254-4034 


\begin{abstract}
In this article, the strategies and techniques used to successfully teach advanced inorganic chemistry, in the lecture and laboratory, to a legally blind student, are described. At Fairfield University, these separate courses, which have a physical chemistry corequisite or a prerequisite, are taught for junior and senior chemistry and biochemistry majors. A student earns a separate grade in both the lecture (3 credits) and the laboratory course ( 2 credits). An overview of the course topics is given, followed by general accommodations and specific approaches that were used. Student assistants were very helpful and provided extra support for the blind student. Student assistants were utilized for the laboratory course, for problem sets, and for exams. Specific examples and detailed explanations of approaches that were helpful to the legally blind student from throughout the entire course are provided. The legally blind student benefited from extensive, verbal description of complexes, figures, and diagrams. In addition, the student benefited from tactile description of figures and models. The student assistants and extra office hours were essential for the blind student to succeed and excel in Advanced Inorganic Chemistry. The approaches discussed in this paper are the product of immediate and continual feedback from the student over the course of the semester. The student would frequently comment after class that he followed the lesson or was confused, and the latter comment elicited experimenting with other approaches.
\end{abstract}




\section{Keywords}

Upper-Division Undergraduate

First-Year Undergraduate/General

Second-Year Undergraduate

Graduate Education/Research

Inorganic Chemistry

Laboratory Instruction

Collaborative/Cooperative Learning

Coordination Compounds

MO Theory

Group Theory/Symmetry 


\section{Introduction}

The visual observations and representations often used to explore and explain chemical phenomena must be translated for a legally blind student to comprehend and appreciate the ideas being demonstrated or discussed. The blind student is a co-author of this manuscript. Several recent articles have stressed the importance to make science learning accessible to blind students or students who have low vision. ${ }^{1-4}$ In a sense, the translation of topics to a blind student is akin to a classic vaudeville act in which an assistant, beholding an object, describes it in such a way that the blindfolded entertainer can positively identify it. As in this act, visual chemical information must be encoded (primarily verbally) so the blind student can form an accurate picture in his/her mind's eye.

This 'translation topic' has not seen much attention in the literature to date. Some of the articles are dedicated toward laboratory adaptations. ${ }^{5-14}$ There have been several recent articles that address using tactile representations to make chemistry more accessible to blind and low vision students. ${ }^{15-16}$ In addition, Tantillo and co-workers reported accommodations that have been made in a computational-theoretical chemistry laboratory for blind students. ${ }^{17}$ Pereira and co-workers reported using sonified infrared spectra and how they were interpreted by blind and low vision students. ${ }^{18}$ Pereira and coworkers also reported a web portal, which blind students can use to process molecular structures. $^{19}$

Those that address the difficulty of making lectures accessible are generally dedicated to logistical, as opposed to content-specific matters (e.g. the need to provide course materials in alternate formats, or to allow sufficient time for their acquisition). ${ }^{16,17}$ 20,21, A notable example is Cary Supalo's report of strategies for the classroom and 
laboratory, as their use to understand specific chemical topics is not detailed. ${ }^{22}$ Two important exceptions are Boyd-Kimball's report on instructional aids developed for a general chemistry course for non-majors, and Harshman et al.'s exploratory study of helpful representations of gas laws at the introductory level. ${ }^{8,20}$ These examples, however, and others ${ }^{15-19}$ underscore the absence of reports on content-specific, fieldtested accommodations in more advanced chemistry courses.

In this article, the strategies and techniques used to successfully teach advanced inorganic chemistry, in the lecture and laboratory, to a legally blind student are described. An overview of the course topics is given, followed by general accommodations and specific approaches that were used. While these methods will not work for every legally blind student due to his/her particular, individual capability to mentally manipulate spatial relationships, they constitute suggestions that, for one student at least, proved helpful. The primary need of a legally blind student that our methods address is access to advanced inorganic chemistry concepts. Our methods attempt to make each step in the logical progression from Lewis structures to molecular geometry, symmetry operations, point group assignments, and qualitative molecular orbital diagrams, intelligible to a visually impaired student. We believe that instructors of sighted students will learn from the approaches we followed in the teaching of the legally blind student and could use some of the methods we describe as an alternate way of describing topics.

\section{Course Overview}

At Fairfield University, Advanced Inorganic Chemistry is intended for junior and senior chemistry and biochemistry majors. Students receive a separate grade for the 
lecture ( 3 credits) and laboratory ( 2 credits) courses. The laboratory course, which meets for four-hours each week, is optional, although most students register for the laboratory. The courses have Physical Chemistry lecture and laboratory as a co-requisite or a prerequisite. The course was designed to introduce the interdependence of chemical bonding, spectroscopic characteristics, and reactivity properties of coordination compounds and complexes using the fundamental concept of symmetry. After reviewing atomic and molecular structure, periodic trends, Lewis structures and valence shell electron pair repulsion (VSEPR) theory, symmetry and group theory were introduced. These approaches were used to understand central atom hybridization, ligand group orbitals, and the construction of qualitative molecular orbital (MO) energy diagrams, with a view to interpreting the spectrochemical series. Electronic spectroscopy, as well as the use of correlation and Tanabe-Sugano diagrams were also introduced.

The laboratory course focuses on the syntheses and characterization of coordination and organometallic complexes. For the laboratory portion of Advanced Inorganic Chemistry, students write either extensive laboratory reports or give an oral presentation for each experiment. For all of the laboratory experiments, students work in groups of two or three.

\section{General Accommodations}

A week before the first class, a pre-semester conference was attended by the instructor, student, and the Director of the University's Disability Service Office. It was arranged that the student would actively take notes on a Braille hand-held computer, while also being provided with an electronic version of the instructor's notes either before or after class. The lecture notes were only referred to by the student in cases 
where his own notes were unclear. In those cases, the text of electronic lecture notes was reviewed using the Job Access with Speech (JAWS) screen reader, with Greek symbols having been added to the reader's dictionary by the student previously. When drawings were insufficiently described in the student's notes, they were either explained from the electronic notes by the student's homework assistant, or the student asked about the relevant material during office hours. The student was thereby able to independently and actively engage with lecture material, and at the same time, know that ambiguities, omissions and other problems with the student's notes could be resolved by checking the instructor's version. The laboratory manual, authored by the instructor, was also provided in an electronic format. Handouts were either posted electronically for the course, or given as physical photocopies. Most handouts had been submitted to the university's disability services office and the State of Connecticut's Vocational Rehabilitation Department five months prior to the start of the semester for braille transcription. For the course textbook, the student relied on an audio recording from Learning Alley (formerly recording for the Blind and dyslexic), on which volunteers for the organization read the text and attempted to describe the figures.

The student is legally blind. He has low usable vision in his right eye with an acuity of 20/800 and cannot see out of his left eye. He can see colors, large shapes and sizable objects. He cannot read print, either on the board or in a text book.

Student assistants formed an important support team for the blind student. These assistants were compensated for their services and were recommended by the course instructor beforehand. There was an assistant in the laboratory course, an assistant for the completion of problem sets, and an assistant for completion of exams. Specifically, one 
assistant helped with weekly laboratory experiments, ensuring that pre-lab diagrams were understood, physical manipulations performed, and observations vividly described. The assistant also drew structures and energy diagrams, as directed, on lab reports prepared by the student using the JAWS screen reader application, Microsoft Word and Microsoft Excel. Another assistant helped with the drawing of energy diagrams and chemical structures, under the student's instruction, on weekly problem sets. A third assistant read exams and took down by dictation the blind student's responses, after they had been worked through on his Braille computer. The instructor made molecular models of structures depicted on exams beforehand. The assistants for assignments and exams had not taken the course, but knew chemistry sufficiently well for their roles. The assistants for problem sets and exams were senior biology majors. These two biology majors were strong students, who had the instructor before. There were two senior chemistry and biochemistry majors who had taken the course as juniors and only one was available to assist. She assisted in the laboratory. All these arrangements were set forth at the presemester meeting. Additionally, extended time was offered on lab reports if needed, but the student never utilized this.

\section{Specific Approaches for Course Content}

In the American Chemical Society's publication Teaching Chemistry to Students with Disabilities: A Manual for High Schools, Colleges, and Graduate Programs, instructor's are directed to "Verbally describe or explain charts, diagrams, graphs, and

other information being presented in a visual format." ${ }^{\prime 10}$ However, best practices for how to describe visual concepts or representations (e.g. symmetry properties, character tables, 
molecular orbital diagrams) are not provided for instructors in any known reference. Furthermore, Cary Supalo emphasizes the construction of tactile diagrams over verbal descriptions. ${ }^{15,16}$ The question naturally arises, "When are verbal descriptions or tactile representations more appropriate?" While depending on the student's ability to form mental pictures to an extent, the answer also depends on the quality of the description. It is the aim of this section to illustrate cases in which particular verbal descriptions were effective, and others in which tactile diagrams or molecular models were more useful.

It is worthwhile to note that Cary Supalo usefully extends the advice of the ACS manual to encompass chemical and mathematical expressions, and illustrates how to avoid verbal ambiguities. With these expressions, literalness in one's description is helpful. To read the formula trans- $\mathrm{K}\left[\mathrm{Mn}\left(\mathrm{C}_{2} \mathrm{O}_{4}\right)_{2}\left(\mathrm{H}_{2} \mathrm{O}\right)_{2}\right]$, for example, one could say " $\mathrm{K}$ open bracket $\mathrm{Mn}$ open parentheses $\mathrm{C}_{2} \mathrm{O}_{4}$ close parentheses taken twice open parentheses $\mathrm{H}_{2} \mathrm{O}$ close parentheses taken twice close bracket." This works for a student familiar with the notational convention of subscripting numbers immediately proceeding element symbols. As a general rule, it is also useful to be mindful in avoiding directional pronouns (e.g. this, that, here, there) in descriptions.

\section{Atomic and Molecular Structure}

Understanding the chemistry of coordination compounds and complexes requires learning about the shapes of the d-orbitals. The student knew the shapes of $\mathrm{s}$ and $\mathrm{p}$ orbitals at the start of the course, but those of $d$ orbitals were only vaguely familiar. Learning their shapes was facilitated by styrofoam models that had the $\mathrm{x}, \mathrm{y}$, and $\mathrm{z}$ axes indicated with metal wires. 
The student was also familiar with the concept of Lewis structures. It was sufficient to state which was the central atom, which were terminal, the number of lone pairs on each atom, and the atoms between which there were multiple bonds. In describing the structure of $\left[\mathrm{IO}_{4}\right]^{-}$, for example, the instructor said, "Place I in the center, surrounded by four O connected to it, each O having 3 lone pairs." (figure 1) Then, in explaining the need to minimize formal charges, the instructor continued, "Move one of the lone pairs from an $\mathrm{O}$ to between I and $\mathrm{O}$ to form a double bond." The formal charge of each "singly bonded" and "doubly-bonded" O was then calculated, and the process was repeated two more times. Each resonance structure was included within brackets, with the charge indicated outside at the upper right.

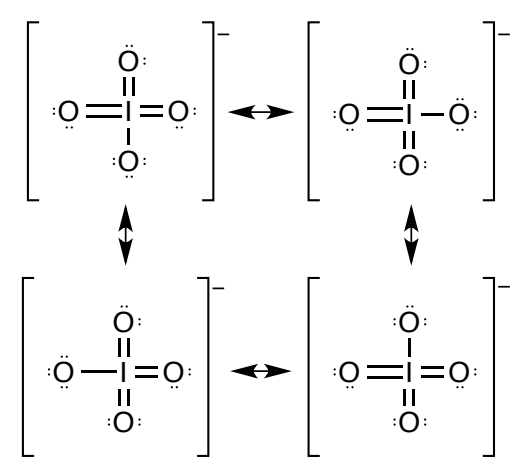

Figure 1. Lewis Structures for $\left[\mathrm{IO}_{4}\right]^{-}$.

Two particularly challenging to describe molecular structures, the porphyrin and corrin ring systems, were encountered in a pre-lab lecture for an experiment designed to synthesize and characterize a Vitamin-B12 model complex (figure 2). Though descriptions were attempted during the lecture, the student was unable to follow them. Consequently, the assistant methodically described the structures after the talk, while the 
instructor helped teams of students start the experiment. The verbal description for Porphyrin is reproduced here.

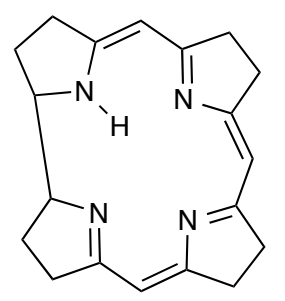

(a)

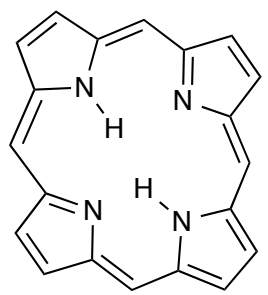

(b)

Figure 2: Corrin (a) and Porphyrin (b) ring

"Imagine a square, and at each corner of the square is a pentagon. Each pentagon consists of four $\mathrm{Cs}$ and one $\mathrm{N}$. The $\mathrm{N}$ of each pentagon points towards the center of the square, and will be referred to as the \#1 position in each pentagon. The others will be numbered in a clockwise fashion. For the pentagon in the upper left hand corner of the macrocylic molecule, there is a double bond at the base of the pentagon, between positions 3 and 4. Position 2 has a substituent that we'll return to. Position 5 is doubly bonded to a $\mathrm{CH}$, which in turn, is singly bonded to the second position of the pentagon in the upper right hand corner of the molecule. In this pentagon, The $\mathrm{N}$ is doubly bonded to position 2 . There is again a double bond between positions 3 and 4 , and a double bond from position 5 to a bridging $\mathrm{CH}$ that connects to the lower right hand corner pentagon at position 2. This pentagon is different. It has double bonds between positions 2 and 3 , and 4 and 5 . Position 5 is singly bonded to a $\mathrm{CH}$, which is doubly bonded to position 2 of the lower left hand corner pentagon. This fourth pentagon has a double bond between positions 3 and 4, like the first 
two. It also has a double bond between positions 1 , the $\mathrm{N}$, and 5. A single bond leads from position 5 to the fourth and final bridging $\mathrm{CH}$ in the molecule, which is doubly bonded to position 2 of the first pentagon at the upper left hand corner. This $\mathrm{CH}$ connecting the fourth to the first pentagon was the substituent that was originally skipped over. Finally, the $\mathrm{N}$ of the first and third pentagons were protonated."

A total of 298 words were used to describe a porphyrin ring. In general, a chemical structure has a lot of meaning.

This description was accompanied by the student attempting to trace in air the molecule as it was explained position-by-position. Movement helped the student picture the molecule mentally, and also permitted the assistant to correct or confirm the student's understanding of the description. Once the structure of porphyrin was understood, that of corrin was much easier to explain. Though the position of double bonds was explained in a similar manner as that for porphyrin, it was only necessary for purposes of the experiment for the assistant to describe the corrin ring system as (I) lacking the fourth bridging $\mathrm{CH}$ group, having instead a direct single bond between position 5 of the fourth pentagon and position two of the first one, and (II) being less unsaturated than porphyrin. Since each description is rather lengthy, understanding one of the structures, and then contrasting it with the other, was the most effective approach. The contrasting was accomplished by comparing the text descriptions.

When molecular geometry was considered, molecular models were often useful to understand the basic shapes predicted by VSEPR theory. Since the student was familiar with an analogue clock, simple shapes, such as trigonal and square planar, could 
be verbally described using the face of a clock as a frame of reference. For example, "BF 3 is trigonal planar, meaning that it has a boron at the center, with fluorine's at the 12,4 , and 8 o'clock positions." For more complex shapes, such as trigonal bipyramidal and octahedral, molecular models were needed. They are particularly convenient for considering possible, alternate structures; for example, whether none, one or both of the lone pairs on $\mathrm{Cl}$ in $\mathrm{ClF}_{3}$ should reside in an equatorial position. In this case, a model of each possible structure was built using a giant-sized, ball-and-stick model kit that consisted of differently colored spheres for atoms and lobes for lone pairs. Each model was shown to the class, and when presented to the blind student, held over his desk directly in front of him, or simply handed to him. The student sat in the front row, in a position illuminated by panels of fluorescent lights on either side. With his limited perception of light, color and shapes, this meant that models held aloft to show the class on either side of his desk were mostly obscured by glare. The tactile representation of each molecule allowed the student to count the number of bonding -pair-bonding pair, lone pair-lone pair, and bonding pair-lone pair interactions. Comparison of these counts recorded on his Braille computer allowed for correct identification of the observed structure.

\section{Types of Coordination Complex Isomerism}

Molecular models, furthermore, were essential for understanding stereo or configurational isomers (e.g. cis versus trans, mer versus fac). They were useful in finding all the possible isomers for a given complex, and which of these were chiral. The 
procedure used for this operation was also helpful, and is illustrated for the complex $\left[\mathrm{CoBrCl}\left(\mathrm{H}_{2} \mathrm{O}\right)_{2}\left(\mathrm{NH}_{3}\right)_{2}\right]^{+}$.

First, the ligands were assigned letters, such that $\mathrm{H}_{2} \mathrm{O}$ was called $\mathrm{A}, \mathrm{NH}_{3}, \mathrm{~B}, \mathrm{Br}$, $\mathrm{C}$, and $\mathrm{Cl}, \mathrm{D}$. A given isomer was specified by indicating which pairs of ligands were trans to each other. For example, $\mathrm{AABBCD}$ meant that the two waters (AA) were trans to each other, the two ammonias (BB) were trans to each other, and the $\mathrm{Br}(\mathrm{C})$ and $\mathrm{Cl}(\mathrm{D})$ ligands had a trans configuration. Different isomers in this case are AABCBD, ACADBB, ABACBD, ABABCD, and ABADBC. This notation, while generally convenient, was especially so for the blind student, because it provided a way to nonvisually record an isomer in his notes so that he could build the model at a later time in the course of studying. For models in- and outside of class, the student benefited from the differently colored parts of the model kit. As long as a convention was specified to or by the student (e.g. ligated waters are represented with white balls), there was no confusion. Before chirality was defined in terms of certain point groups in the course, the student occasionally built a model and its mirror image to resolve whether the molecule was chiral. As the student became more comfortable with identifying the presence or absence of symmetry elements, however, this was mostly unnecessary.

In contrast to the need of models to examine stereoisomers, examples of ionization, solvent, coordination, and linkage isomers were all adequately handled by explicitly reading chemical formulas, as illustrated previously.

\section{Crystal Field Theory}


The orientation of the d-orbitals was first reviewed by emphasizing that three $\left(\mathrm{d}_{\mathrm{xy}}\right.$, $d_{x z}$ and $\left.d_{y z}\right)$ had lobes between axes, and the other two $\left(d_{x-y}^{2}{ }^{2}\right.$ and $\left.d_{z}^{2}\right)$, on them. The torus of $\mathrm{d}_{\mathrm{z}}^{2}$ in the xy-plane was also noted. This preface made the following geometric explanation of Crystal Field Theory (CFT) understandable to the blind student.

A free metal ion was envisioned, with its five degenerate d-orbitals at a certain energy. The accompanying energy diagram for the d-orbitals was described as consisting of five orbitals, all at the same level. The metal ion was assumed to have an $\mathrm{n}^{+}$charge, and it was imagined that a spherical charge of $6^{-}$was then applied. All five orbital slots on the diagram were said to rise in energy. The next step was to imagine, instead of a spherical field, the presence of negative point charges at the opposite ends of the $\mathrm{x}, \mathrm{y}$, and $\mathrm{z}$ axes. Three slots, representing the d-orbitals orientated between axes, were said to move to lower energy, while the remaining two, with lobes on the axes, rose in energy. Though the symmetry unit had not yet been covered, the triply and doubly degenerate sets of d-orbitals were labeled $\mathrm{T}_{2 \mathrm{~g}}$ and $\mathrm{E}_{\mathrm{g}}$, respectively, for ease of reference (figure 3).

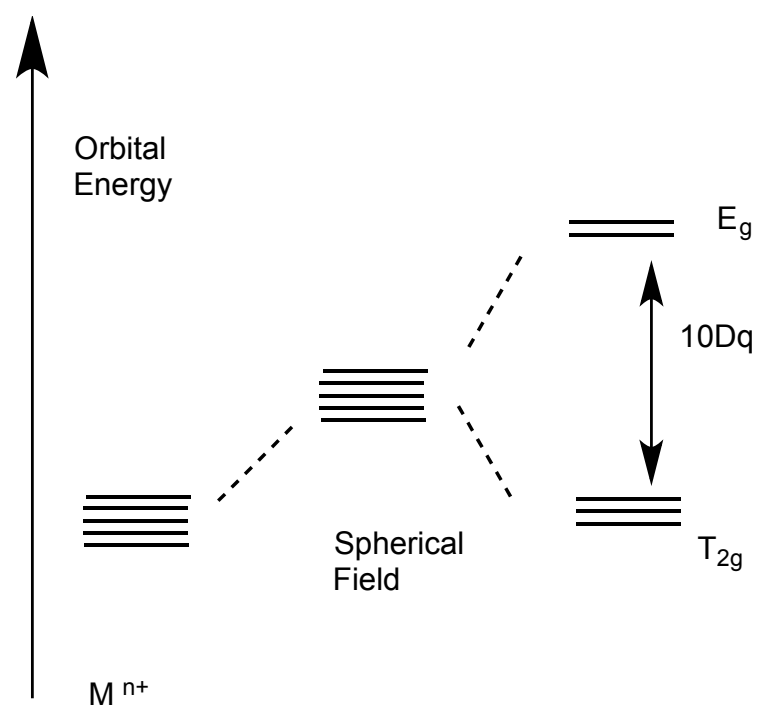

Figure 3. Crystal Field Theory and d-orbital splittings in a complex with octahedral symmetry. 
The difference between them was defined as $10 \mathrm{D}_{\mathrm{q}}$. The position of the $\mathrm{d}$ orbitals in a spherical field (the result of the second step in this procedure) was labeled the bary center. It was initially difficult for the blind student to reconcile the description of the $T_{2 g}$ set as being closer to the bary center than the $\mathrm{E}_{\mathrm{g}}$ set, with the statement that the amount of stabilization and destabilization canceled one another. This was resolved by explaining that each of the three $T_{2 g}$ orbitals was $-0.4 \Delta_{o}$ below the bary center, and each of the two $E_{\mathrm{g}}$ set was $0.6 \Delta_{\mathrm{o}}$ above it, meaning that either set differed by a total of $1.2 \Delta_{\mathrm{o}}$ from the bary center.

The d orbital electron configurations for the $2^{+}$and $3^{+}$oxidation states of $\mathrm{Ti}, \mathrm{Cr}$ and Fe were illustrated using the energy diagram thus derived. The examples, covering $\mathrm{d}^{1}$ through $\mathrm{d}^{6}$ configurations, were used to systematically add electrons. The configurations were described by stating how many of the $\mathrm{T}_{2 \mathrm{~g}}$ and/or $\mathrm{E}_{\mathrm{g}}$ slots contained "one" or "a pair." For example, the high spin $\mathrm{d}^{5}$ configuration was described as "one in each of the orbitals," whereas the $\mathrm{d}^{5}$ low spin state had "two paired sets and one unpaired in $\mathrm{T}_{2 \mathrm{~g}}$." With this information, the student was able to visualize the number of electrons in the $T_{2 g}$ and $E_{g}$ sets, the number of pairing interactions, and the possible electronic exchanges. As a result, the blind student was able to express the total energy of a given d- electron configuration as the sum of the crystal field stabilization energy, exchange energy, and pairing energy. Knowing the number of unpaired electrons, the student was also able to calculate the effective magnetic moment, the formula for which had been read explicitly as explained earlier. 
CFT d-orbital splitting diagrams for tetrahedral and square planar complexes were treated in much the same fashion. For the former, the negative point charges were envisioned at opposite corners of a cube, coming closer to the d-orbitals directed towards the cube's edges than those with lobes lying on the axes. The orbital overlap for either set was said to be not as direct as for the octahedral complex, thereby explaining in part the smaller energetic splitting of the d-orbital sets. For the latter, the point charges on the zaxis of the octahedral complex were envisioned as being pulled away, causing the dorbitals containing a z-component to fall in energy. For both cases, the orbital slots on the diagrams were labeled, and read sequentially from lowest to highest energy.

\section{Symmetry and Group Theory}

Again, molecular models played a pivotal role in demonstrating the elements of symmetry to the blind student, who, prior to the course, found the relevant chapter in the textbook incomprehensible. It was generally helpful to hold the molecule in one hand directly in front of the student, while indicating the location of a mirror plane or inversion center with the other. Axes of rotation were shown by rotating the molecule about each by the hand that held the model. A given symmetry property was either first shown to the class or to the blind student so as to not give any additional special attention to the student.

The most difficult symmetry element to visualize was an improper axis of rotation. The example given to the class was the $S_{4}$ axis of methane and is given in figure 4. Though manipulation of a model would perhaps have been the easiest approach to demonstrate this property, a verbal description was attempted. "picture a tetrahedron 
inscribed in a cube. Spheres at opposite corners of a cube-front upper left and back upper right, back lower left and front lower right—-form a tetrahedron. Number these spheres 1 through 4 , respectively. If the molecule is rotated by $90^{\circ}$ and then inverted, so the spheres at the bottom of the cube are brought upstairs and vice-versa, the molecule in its original orientation is reproduced."
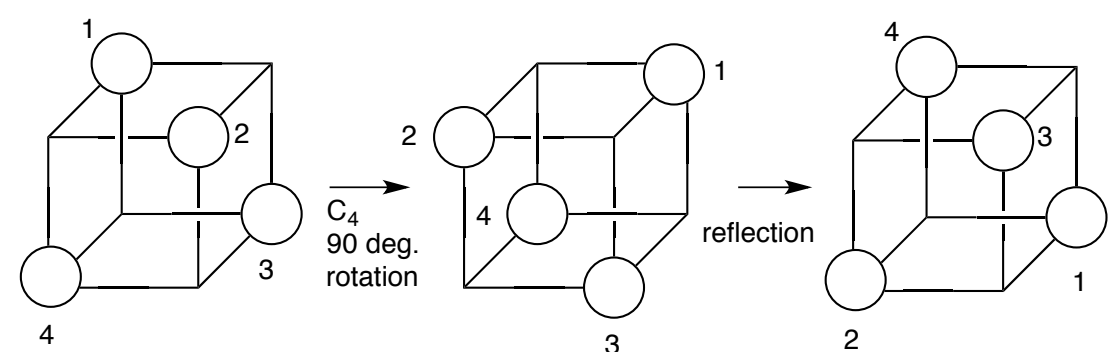

Figure 4. Tetrahedron inscribed in a cube to represent a $\mathrm{S}_{4}$ axis.

Notwithstanding the clarity of this description, it was helpful for the student to come to the board after class. His index finger (with guidance) was used to trace the dimensions of the cube, and to point out the numbering pattern. Then the $90^{\circ}$ rotation was illustrated by positioning two of the student's fingers at opposite corners, and indicating to which corner each finger would move. The same approach was used to show the outcome of the reflection operation.

Second only to the usefulness of molecular models for the blind student to visualize the symmetry properties of a molecule was exhaustive practice with models on his part.

Point groups of molecules were assigned by examining molecular models according to a flow chart that was presented during lectures as a series of questions about the presence of certain symmetry elements. 
When character tables were discussed, the column headings, the characters of the irreducible representations (in rows), and the corresponding information about orbitals were read row-by-row.

\section{Molecular Orbital Theory}

Molecular orbital (MO) theory was explained through the linear combination of atomic orbitals method to form bonding, anti-bonding, and non-bonding orbitals. Each was described based on its effect on electron density in the internuclear region. The simplest case was the addition of "two spherical s-orbitals of positive symmetry" to form "a bigger, oval lobe of positive symmetry," and their subtraction to yield "spherical lobes of opposite symmetry separated by a node." (figure 5). 


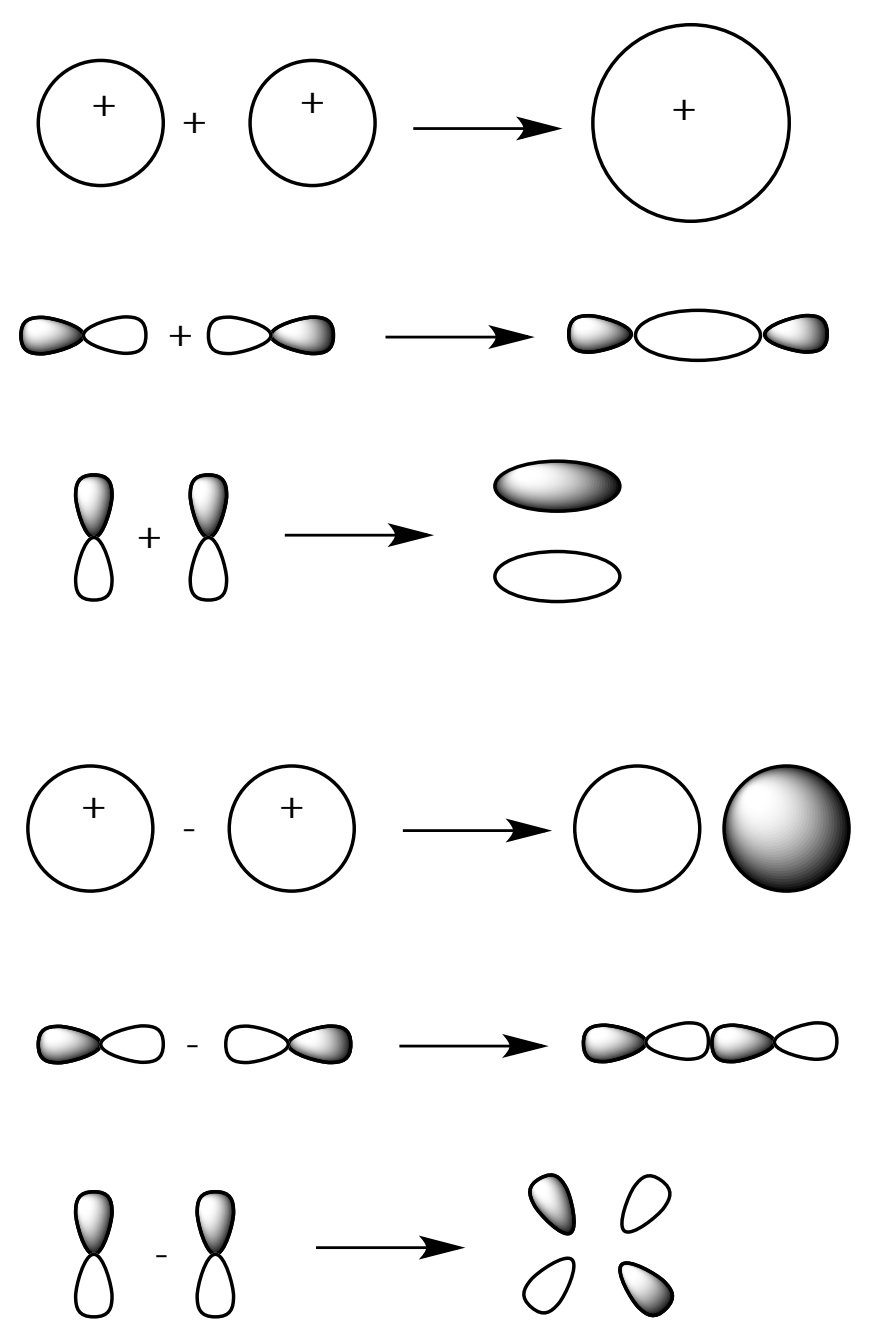

Figure 5. Linear combination of atomic orbitals showing both bonding and antibonding orbitals.

Every change in sign was understood to imply the presence of a node. The addition of two p-orbitals on the internuclear axis was described as "negative positive plus positive negative," and resulted in "two outer negative lobes, and a middle positive lobe roughly double in size." Their subtraction yielded a four lobed anti-bonding orbital described as "negative positive negative positive." Combination of either pair of the perpendicular p-orbitals was described similarly. For example, one set was drawn 
vertically and described as "positive-negative, top-bottom for each." The resulting bonding orbital consisted of "a positive lobe on top, negative on bottom," and the antibonding, "positive-negative, top-bottom on the left, and negative-positive, right."

Description of MO energy diagrams consisted of stating the atomic orbitals on the left- and right-hand sides, the molecular orbitals in the middle, and from and to where tie lines were drawn. Orbital occupancy was read from lowest to highest energy as for CFT d-orbital splitting diagrams. A sense of the spacing in these diagrams was conveyed by stating the orbital energies in electron volts. The description of the $\mathrm{MO}$ diagram for $\mathrm{O}_{2}$ is given as an example (figure 6) 


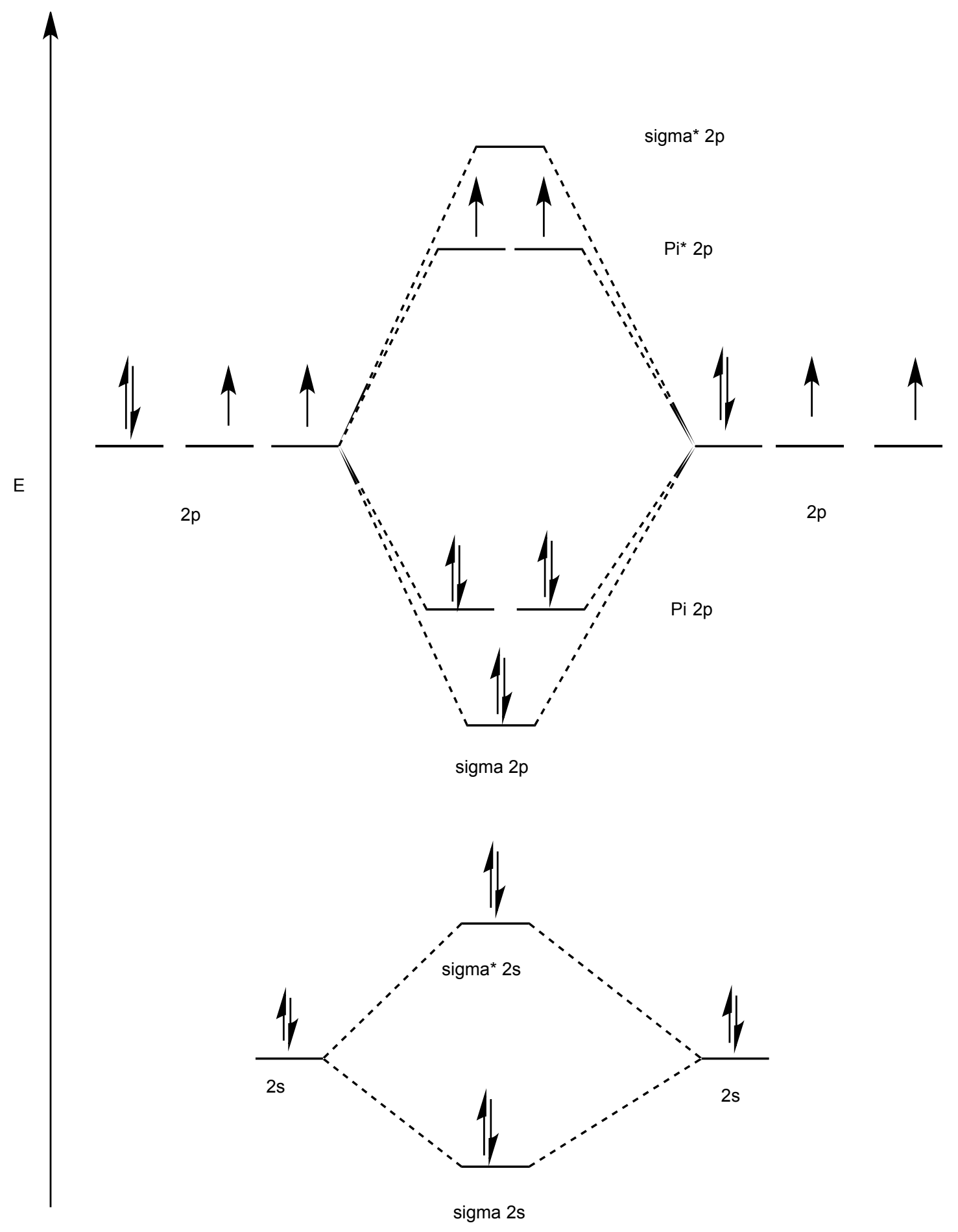

Figure 6. Molecular Orbital diagram for $\mathrm{O}_{2}$. 
Atomic orbitals for oxygen are on the left and right. On each side, there is one $2 \mathrm{~s}$ atomic orbital at $-32.38 \mathrm{eV}$ and above it, three $2 \mathrm{p}$ orbitals at -15.58 $\mathrm{eV}$. Both $2 \mathrm{~s}$ orbitals interact, giving sigma(2s) in the middle beneath their level, and a sigma*(2s), above their level. The vertical or energetic difference between $\operatorname{sigma}^{*}(2 \mathrm{~s})$ and the $2 \mathrm{~s}$ orbitals on either side is greater than that between them and sigma(2s). Tie lines connect the $2 \mathrm{~s}$ orbitals to these MOs. In addition, one $2 \mathrm{p}$ orbital from the left and right interact to form sigma( $2 p)$ below their vertical position. The other two $2 p$ orbitals from each side form two $\mathrm{Pi}(2 \mathrm{p})$, below, and two $\mathrm{Pi}^{*}(2 \mathrm{p})$, above their level. The $p$ orbitals that formed sigma $(2 p)$, also form sigma*(2p), which is the highest energy MO. As before, tie lines connect the atomic orbitals to the bonding and anti-bonding molecular orbitals they form. The electron configuration on each side is "two s two, two p four." For the MOs in the middle, it is "sigma two s two, sigma star two s two, sigma two $p$ two, pi two $p$ four, with a pair in each of the two degenerate orbitals of this kind, and pi star two p two, with one up in each degenerate orbital of this kind."

Once the energy ordering of the molecular orbitals for a given system is clearly stated, an alternative way to summarize their occupancy is by identifying the HOMO and the LUMO, and the number of electrons in the former. 
For systems other than homo- or heteronuclear diatomics, the method of constructing ligand group orbitals by inspection was employed, as described for methane (figure 7).

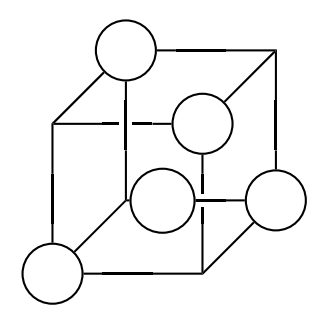

LGO $a_{1}$

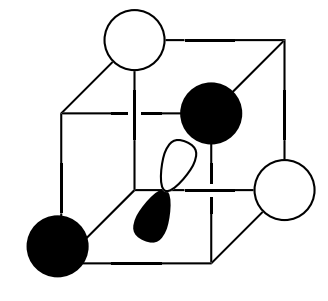

$\mathrm{LGO} \mathrm{t}_{2}$

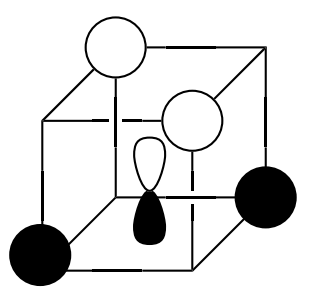

LGO $t_{2}$

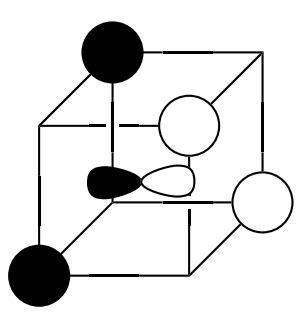

$\mathrm{LGO} \mathrm{t}_{2}$

Figure 7. Ligand Group Orbitals and their overlap with the atomic orbitals of carbon.

Draw four cubes. At their center, put an $\mathrm{s}^{-}$or $\mathrm{p}_{\mathrm{x}^{-}}$or $\mathrm{p}_{\mathrm{y}^{-}}$or $\mathrm{p}_{\mathrm{z}^{-}}$orbital. Axes: $\mathrm{z}$ vertical, $\mathrm{y}$, left-to-right, and $\mathrm{x}$, into-and-out of the board. The positive symmetry part of the $\mathrm{p}_{\mathrm{x}}$ orbital is, open towards you, and the hatched or negative symmetry part behind. The positive symmetry part of the $\mathrm{p}_{\mathrm{y}}$ is open to the right, and the negative symmetry part on left. The positive symmetry part of the $\mathrm{p}_{z}$ is open up, and the negative symmetry part is down. Numbered corners: upper right-hand ligand group orbital (LGO), 1, lower right-hand LGO 2, lower left-hand LGO 3, upper left LGO 4. LGOs1 and 3 form face of the cube facing class; LGOs 2 and 4 form the other face going into the board. For overlap with $p_{x}$, LGOs 2 and 4 need negative symmetry; LGO's 1 and 3, require positive symmetry phasing. For $p_{y}$ overlap, LGOs 3 and 4 require negative symmetry, and LGOs 1 and 2 require positive symmetry. For $\mathrm{p}_{\mathrm{z}}$ 
overlap, LGOs 1 and 4 require positive symmetry and, LGOs 2 and 3, require negative symmetry.

For the MO diagram (figure 8): the $2 \mathrm{~s}(-19.43 \mathrm{eV})$ and $2 \mathrm{p}(-10.66 \mathrm{eV})$ orbitals from the central $\mathrm{C}$ were drawn on the left and the 1s LGOs from $\mathrm{H}(-13.6 \mathrm{eV})$ were drawn on the right. The $2 \mathrm{~s}$ orbital from $\mathrm{C}$ interacts with the $1 \mathrm{~s}$ orbital from $\mathrm{H}$ to form molecular orbital $\mathrm{A}_{1}$. The three $2 \mathrm{p}$ orbitals on $\mathrm{C}$ interact with the LGOs from $\mathrm{H}$ to give a degenerate set of three $\mathrm{T}_{2}$ molecular orbitals. The next MO is $\mathrm{A}_{1}{ }^{*}$, which comes from $2 \mathrm{~s}$ on the left and $1 \mathrm{~s}$ on the right, and the highest energy $\mathrm{MO}$ is $\mathrm{T}_{2}^{*}$, which comes from the $2 \mathrm{p}$ orbitals on the left and the 1s LGOs of $\mathrm{H}$. The HOMO is $\mathrm{T}_{2}$, with three pairs, and the LUMO is $\mathrm{A}_{1}$ * 


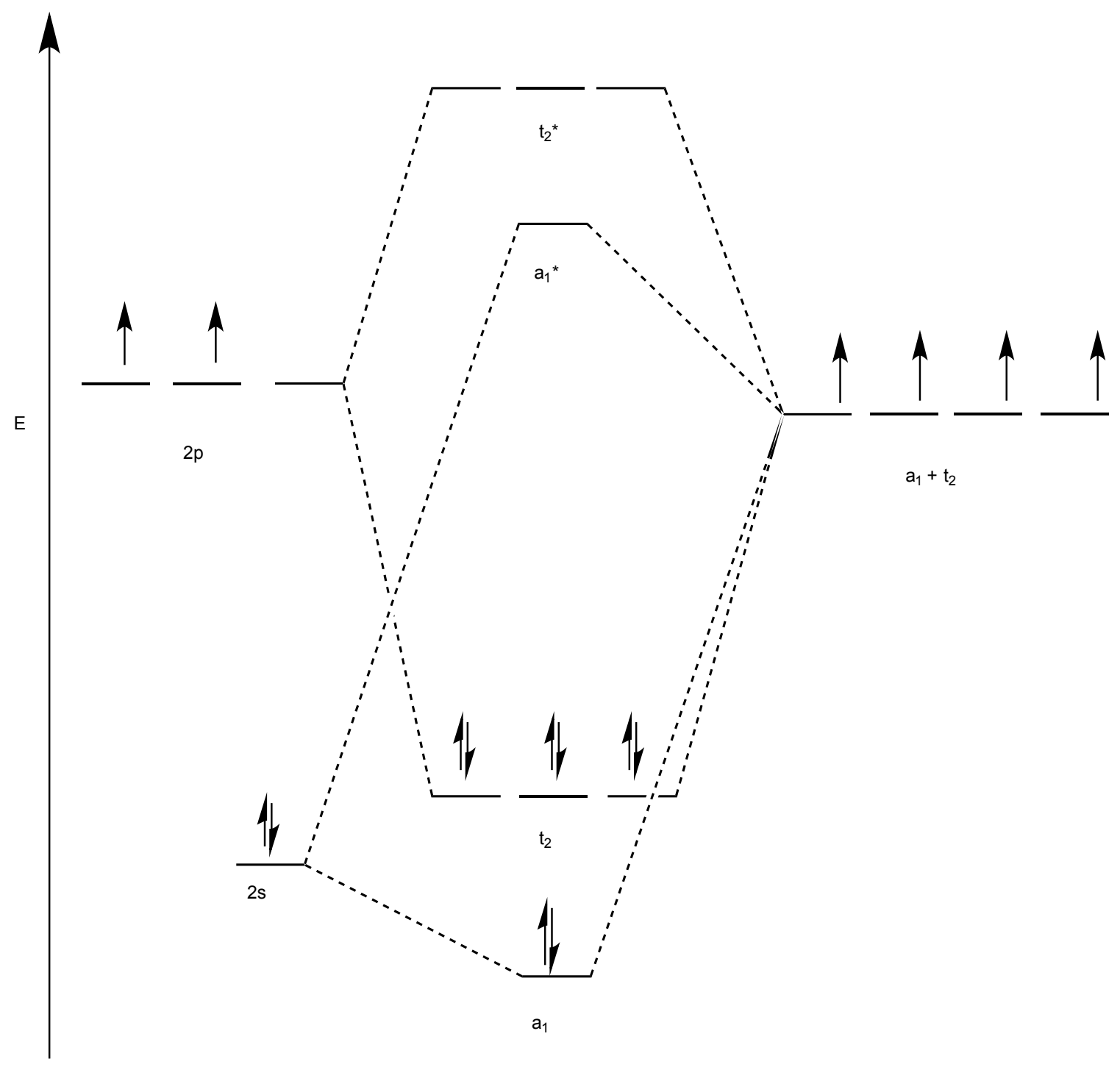

Figure 8. Qualitative $\mathrm{MO}$ diagram for $\mathrm{CH}_{4}$.

The approach used for methane was extended to explain pi-acceptor, sigmadonor, and pi-donor octahedral complexes. The atomic orbitals on the central metal ion were drawn in boxes, in an analogous fashion to those of $\mathrm{C}$ in methane. For this case, however, the axes were defined somewhat differently. The z-axis was said to be vertical, with the top labeled position 1 and the bottom, 6. The y-axis extended from the bottom right, labeled position 3, to top left, labeled 5. The x-axis extended from the top right, position 2, to bottom left, position 4. The construction of LGOs, and the MO diagrams, 
were otherwise described similarly. It was helpful for the instructor to compare the relative size of the HOMO-LUMO gap among the three MO diagrams for octahedral complexes, in order to explain the ordering of the spectrochemical series.

\section{Tanabe-Sugano diagrams}

MO diagrams were used as a starting point for understanding electronic spectra. In addition, Tanabe-Sugano diagrams were utilized to interpret UV-Visible spectra for first-row octahedral metal complexes. The following steps were helpful for the blind student in interpreting Tanabe-Sugano diagrams:

- Read the ground state, then the excited states of the same multiplicity

- Enumerate each transition, from lowest to highest energy, by stating the ground state and then a given excited state.

- Read the energy of the transitions from spectral data

- Describe drawing a vertical line at a test value on the $\mathrm{Dq} / \mathrm{B}$ axis that intersects with each of the curves of the same multiplicity as the ground state.

- Describe drawing horizontal lines from the points of intersection to get their ycoordinates $(\mathrm{E} / \mathrm{B})$.

- Calculate the ratio of the y-values for a given transition. Compare each to the ratios found from the spectral data.

- This method was easy for the blind student to follow in class, and work through on an exam. (e.g. if the blind student asked reader, "If you draw a line at Dq/B = 2, where about does it intersect curves with a multiplicity of 4?" The reader would say, "it intersects $4 \mathrm{~T}_{2}$ at 19, , and so on.) 


\section{Laboratory Considerations}

The laboratory course was designed as a synthetic inorganic chemistry laboratory with an emphasis on characterization. The students prepare a variety of coordination and organometallic complexes including one air sensitive complex.

The blind student prepared his notebook using Microsoft Word before the laboratory. This entry included a bullet-point version of the procedure with calculated quantities for reagents. In the laboratory, the physical manipulations and measurements were accomplished by other group members or the laboratory assistant. The solutions were prepared and placed in the instruments (UV-Vis spectrometer, NMR spectrometer, magnetic susceptibility balance, polarimeter, or ATR-IR spectrometer) by other laboratory group members or the laboratory assistant.

While in the laboratory, the student provided direction to the assistant. The student was allowed to provide as much direction as possible. Usually, this consisted in conveying the instructions from the procedure that the student had outlined in his notebook prior to the experiment. The student, for example, provided instruction as to the quantities of reagents, and the type of glassware to be used. Meanwhile, the assistant provided observational feedback to the student as to what was transpiring in the laboratory. Observation of color changes during the course of reactions was critical to the laboratory experience. The assistant provided vivid and detailed descriptions of these phenomena. An example of this is given below for the preparation of pentaamminechlorocobalt(III) chloride, which was synthesized in the laboratory.

$\mathrm{CoCl}_{2} \cdot 6 \mathrm{H}_{2} \mathrm{O}(10.001 \mathrm{~g}, 42.033 \mathrm{mmol})$ was added to a solution in which $\mathrm{NH}_{4} \mathrm{Cl}$ (5.0136, $93.727 \mathrm{mmol}$ ) had been dissolved with stirring in $30 \mathrm{~mL}$ of $\mathrm{NH}_{4} \mathrm{OH}$. The 
resulting brownish-pink solution, which contained some solid material, transitioned to a blackish-pink, or raspberry jelly color, with some bubbling, upon the dropwise addition of $10 \mathrm{~mL}$ of $\mathrm{H}_{2} \mathrm{O}_{2}$ over a period of ten minutes. Subsequent addition of $30 \mathrm{~mL}$ of concentrated $\mathrm{HCl}$ to this solution resulted in the vigorous evolution of gas and heat, and a wine-colored solution. After heating the reaction mixture for 20 minutes at $85^{\circ} \mathrm{C}$, the solid present in the solution changed from rose to deep lilac in color. The mixture was then cooled to room temperature, and gooey, purple crystals were collected on a Buchner funnel from an indigo solution.

Feedback to the student was frequent and continual, assuring that the student knew what the assistant was doing and observing, unless the assistant needed to have their undivided attention on the task at hand. At these times, the student was informed that the flow of descriptive feedback would be stopped for a few moments.

\section{Summary}

This article provides the strategies and techniques used to successfully teach advanced inorganic chemistry, in the lecture and laboratory, to a blind student. Student assistants were very helpful and provided extra support for the blind student. We provided specific examples and detailed explanations from throughout the entire course, that were helpful to the blind student. In general, we have given examples where two dimensional energy diagrams, molecular orbital energy diagrams, nomenclature, and two-dimensional structures were handled well by verbal descriptions. We have also given examples where spatial relationships, three-dimensional structures, and Tanabe-Sugano diagrams were handled well by three-dimensional tactile representations. Instructors of sighted students 
will learn from the approaches we followed in the teaching of the legally blind student and could use some of the methods we described as an alternate way of describing topics.

\section{Acknowledgements:}

The authors are grateful for all of the assistance that the Office of Disability Support Services at Fairfield University provided for the blind student. The authors would like to thank Prof. L. Kraig Steffen for helpful suggestions.

\section{References}

(1) Supalo, C.A.; Mallouk, T.E.; Amorosi, C.; Lanouette, J. Using Adaptive Tools and Techniques to Teach a Class of Students Who Are Blind or Low-Vision. J. Chem. Educ, 2009, 86 (5), 587-591.

(2) Supalo, C.A.; Hill, A.A.; Larrick, C. G. Summer Enrichment Programs to Foster Interest in STEM Education for Students with Blindness or Low Vision.” J. Chem. Educ, 2014, 91, 1257-1260.

(3) Supalo, C.A.; Isaacson, M.D.; Lombardi, M.V. Making Hands-On Science Learning Accessible for Students Who are Blind or Have Low Vision. J. Chem. Educ, 2014, 91, 195-199.

(4) Wedler, H.B.; Boyes, L.; Davis, R.L.; Flynn, D.; Franz, A.; Hamann, C.S.; Harrison, J.G.; Lodewyk, M.W.; Milinkevich, K.A.; Shaw, J.T.; Tantillo, D.J.; 
Wang, S.C. Nobody Can See Atoms: Science Camps Highlighting Approaches for Making Chemistry Accessible to Blind and Visually Impaired Students. J. Chem. Educ, 2014, 91, 188-194.

(5) Tombaugh, D. Chemistry and the Visually Impaired. J Chem. Educ, 1981, 58 (3), 222-226.

(6) Flair, M.N.; Setzer, W.N. A Laboratory Technique for the Visually Impaired. $J$. Chem. Educ., 1990, 67(9), 795-796.

(7) Supalo, C.A.; Mallouk, T.E.; Rankel, L.; Amorosi, C.; Graybill, C.M. Low-cost Laboratory Adaptations for Precollege Students who are Blind or Visually Impaired. J. Chem. Educ, 2008, 85 (2), 243-247.

(8) Boyd-Kimball, D. Adaptive Instructional Aids for Teaching a Blind Student in a Nonmajors College Chemistry Course. J. Chem. Educ. 2012, 89 (11), $1395-1399$.

(9) Hiemenz, P. C.; Pfeiffer, E. A General Chemistry Experiment for the Blind. J. Chem. Educ., 1972, 49 (4), 263.

(10) Tallman, D.E. $\mathrm{pH}$ Titration Apparatus for the Blind Student. J. Chem. Educ., 1978, 55 (9), 605.

(11) Lunney, D.; Morrison, R. C. High Technology Laboratory Aids for Visually Handicapped Chemistry Students. J. Chem. Educ., 1981, 58 (3), 228.

(12) Anderson, J. L. Chemical Instrumentation for the Visually Handicapped. J. Chem. Educ., 1982, 59 (10), 871.

(13) Clauss, A. JCE Concept Collections: Making Chemistry Accessible. J. Chem. Educ., 2009, $86(5), 591$. 
Ratliff, J.L. Chemistry for the Visually Impaired. J. Chem. Educ., 1997, $74(6), 710-711$.

$$
\text { Supalo, C.A.; Kennedy, S.H. Using Commercially Available Techniques }
$$

to make Organic Chemistry Representations Tactile and More Accessible to Students with Blindness or Low Vision. J. Chem. Educ, 2014, 91 (10), $1745-$ 1747.

$$
\text { Poon, T.; Ovadia, R. Using Tactile Learning Aids for Students with }
$$

Visual Impairments in a First-Semester Organic Chemistry Course. J. Chem.

Educ, 2008, 85 (2), 240-242.

Wedler, H.B.; Cohen, S.R.; Davis, R.L.; Harrison, J.G.; Siebert, M.R;

Willenbring, D.; Hamann, C.S.; Shaw, J.T.; Tantillo, D.J. Applied Computational Chemistry for the Blind and Visually Impaired, J. Chem. Educ, 2012, 89, 14001404.

(18) Pereira, F.; Ponte-e-Sousa, J.; Fartaria, R.P.S.; Bonifácio, V.D.B.; Mata, P.; Aires-de-Sousa, J.; Lobo, A.M. Sonified Infrared Spectra and Their Interpretation by Blind and Visually Impaired Students. J. Chem. Educ, 2013, 90, 1028-1031.

$$
\text { Pereira, F.; Aires-de-Sousa, J.; Bonifácio, V.D.B.; Mata, P.; Lobo, A.M. }
$$

MOLinsight: A Web Portal for the Processing of Molecular Structures by Blind Students. J. Chem. Educ, 2011, 88(3), 361-362.

(20) Harshman, J.; Bretz, S. L.; Yezierski, E. Seeing Chemistry through the Eyes of the Blind: A Case Study Examining Multiple Gas Law Representations. J. Chem. Educ., 2013, 90 (6), 710-716. 
(21) Milner, D.; Nieman, R.; Swanson, A.; Woods, M.; Carpenter, K. Teaching Chemistry to Students with Disabilities: A Manual for High Schools, Colleges, and Graduate Programs, 4th ed.; American Chemical Society: Washington, DC, 2001.

(22) Supalo, C. Techniques To Enhance Instructors' Teaching Effectiveness with Chemistry Students Who Are Blind or Visually Impaired. J. Chem. Educ., 2005, 82 (10), 1513-1538. 